\title{
Towards Accurate and Reproducible Uranium Isotopic Analysis via Atom Probe Mass Spectrometry
}

\author{
Frederick Meisenkothen ${ }^{1}$, Mark McLean ${ }^{1}$, Irina Kalish ${ }^{2}$, Daniel Samarov $^{1}$ and Eric Steel ${ }^{1}$ \\ ${ }^{1}$ National Institute of Standards and Technology, Gaithersburg, Maryland, United States, ${ }^{2}$ MATSYS, Inc., \\ Sterling, Virginia, United States
}

Atom probe tomography (APT) is increasingly being used to perform sub-micrometer-scale isotopic analyses in geological, nuclear, semiconductor, and meteoritic materials. APT offers several advantages over other commonly used mass spectrometry techniques (e.g., secondary ion mass spectrometry and thermal ionization mass spectrometry) for analyses in this size regime. The benefits include a combined ionization and detection efficiency that can approach 80\%; an ion-species-independent detection efficiency; and a 3-dimensional chemically- and isotopically-resolved imaging capability. Therefore, APT can theoretically achieve a given level of measurement uncertainty with an analysis volume roughly an order of magnitude smaller than that required by another mass spectrometry technique. However, several studies have shown quantitative analysis results in the atom probe can be inaccurate (Fahey, et al., 2016; Hudson, et al., 2011). We, thus, have focused our efforts on exploring the limits of accuracy in atom probe isotopic analyses and toward finding ways to improve accuracy and reproducibility in our measurements.

We have developed a three-pronged approach to achieve accurate and reproducible isotopic analyses in APT, that combines data filtering, machine learning-based peak fitting, and calibration. Since isotopic analysis is one of the simplest types of measurement we can perform with APT, these analyses provide a basis for assessing the ultimate accuracy of atom probe mass spectrometry. In addition to natural materials, a special set of certified uranium isotopic reference materials was used in the study. By comparing the measured isotopic abundance values to the certified reference values, it was possible to make quantitative statements about accuracy. Figure 1 shows an example of a peak fitting result for the SRM U-900 reference material. The spectrum has been decomposed into three component peaks, each associated with a different isotope that was included in the analysis. The total fit is a linear combination of these three component peaks. Note that the fitting algorithm did an excellent job of capturing the complex tail forms on each peak and properly attributed the hydride peaks to their respective "parent" peaks. Table 1 gives a summary of the isotopic analysis results achieved by our methods, as represented by the percent relative error (percent relative error $=100 \% \mathrm{x}$ [measured value - reference value]/[reference value]). The U-235 and U-238 abundance accuracy was better than $+/-1 \%$ relative error, on average. Further, no single measurement uncertainty was outside the range $+/-1.5 \%$ relative error for these two isotopes. The measurement uncertainty is a significant improvement over results previously reported for similar materials (Fahey, et al., 2016) and is roughly consistent with an uncertainty limited primarily by Poisson counting statistics, i.e., the number of uranium atoms recorded for analysis. 

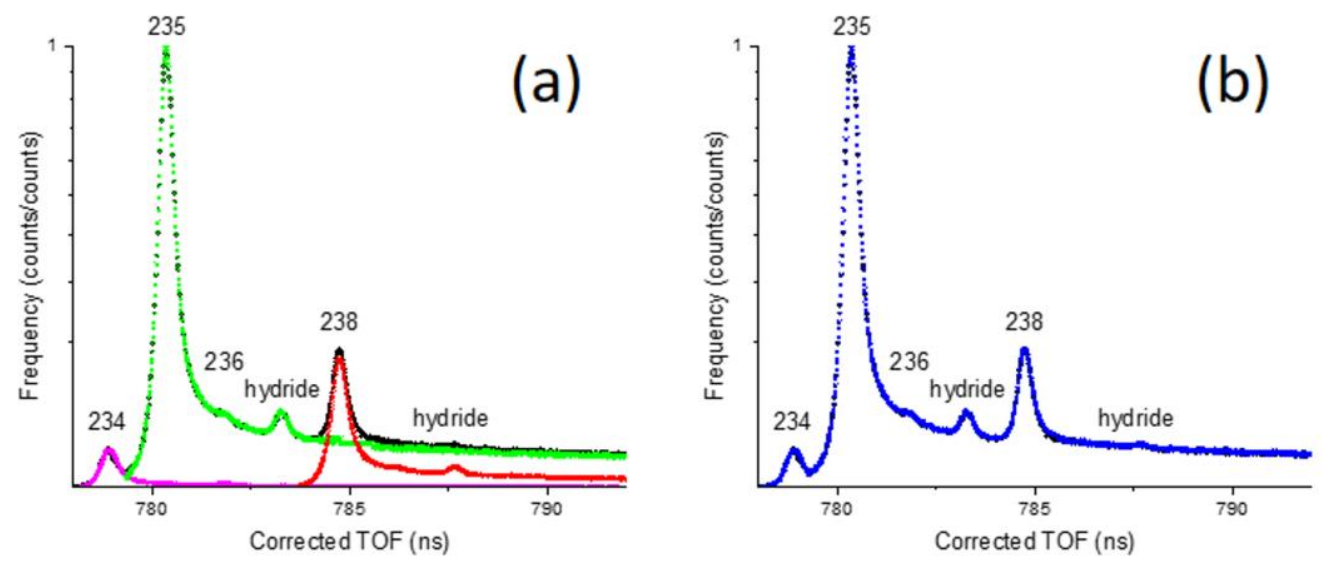

Figure 1. Peak fitting example, SRM U-900. (a) Peaks attributed to each isotope (black = empirical data, magenta $=\mathrm{U}-234$, green $=\mathrm{U}-235$, red $=\mathrm{U}-238$ ). (b) Total fit (blue).

\begin{tabular}{lccc}
\hline $\begin{array}{c}\text { Reference } \\
\text { Material }\end{array}$ & Isotope & $\begin{array}{c}\text { Certified } \\
\text { Abundance }\end{array}$ & $\begin{array}{c}\text { Average } \\
\text { Rel Error }\end{array}$ \\
\hline & & (\%) & $(\%)$ \\
\hline SRM U-030a & 234 & 0.02778 & -6.6 \\
SRM U-350 & 234 & 0.2498 & 1.5 \\
CRM 129-A & 235 & 0.72087 & -0.1 \\
SRM U-900 & 234 & 0.7777 & -0.8 \\
SRM U-030a & 235 & 3.0404 & -0.1 \\
SRM U-900 & 238 & 8.693 & -0.2 \\
SRM U-350 & 235 & 35.19 & 0.2 \\
SRM U-350 & 238 & 64.393 & -0.1 \\
SRM U-900 & 235 & 90.196 & -0.1 \\
SRM U-030a & 238 & 96.9312 & -0.6 \\
CRM 129-A & 238 & 99.27382 & -0.7
\end{tabular}

Figure 2. Table 1: Average uranium isotopic analysis results for four certified reference materials.

\section{References}

Fahey, A.J., Perea, D.E., Bartrand, J., Arey, B.W. \& Thevuthasan, S. (2016). Uranium isotopic ratio measurements of U 308 reference materials by atom probe tomography. Journal of Environmental Radioactivity 153, 206-213.

Hudson, D., Smith, G.D.W. \& Gault, B. (2011). Optimisation of mass ranging for atom probe microanalysis and application to the corrosion processes in $\mathrm{Zr}$ alloys. Ultramicroscopy 111(6), 480-486. 